\title{
WILEY-VCH
}

DOI: 10.1002/ ((please add manuscript number))

Article type: Research News

\section{Carbon nanotubes in perovskite solar cells}

Severin N. Habisreutinger, Robin J. Nicholas, Henry J. Snaith*

Severin N. Habisreutinger, Prof. Robin J. Nicholas, Prof. Henry J. Snaith

Clarendon Laboratory, Department of Physics, University of Oxford, Parks Road, OX1 3PU, Oxford, UK

E-mail: henry.snaith@physics.ox.ac.uk, robin.nicholas@physics.com

Keywords: perovskite solar cells, single-walled carbon nanotubes, multi-walled carbon nanotubes, carbon nanotubes

\begin{abstract}
The remarkable optoelectronic properties of metal halide perovskite absorbers have, in the past years, made the perovskite solar cell one of the most promising emerging photovoltaic technologies. The charge collecting layers are essential parts of this type of solar cell. Carbon nanotubes have emerged as a potential candidate to take on this role. Equipped with a range of highly beneficial properties including excellent charge transport characteristics, chemical inertness, as well as mechanical robustness, carbon nanotubes are able to both efficiently extract photogenerated charges, and improve the resilience and stability of a perovskite solar cell. In this Research News article we give a concise overview of the current state-of-the-art of perovskite solar cells in which carbon nanotubes are incorporated as a charge conduction layer.
\end{abstract}

\section{Introduction}




\section{WILEY-VCH}

Over the last two decades, carbon nanotubes have generated a lot of excitement among researchers involved in the field of material science and engineering, specifically those with a focus on electronic and optoelectronic applications. The cause for the excitement is the truly exceptional charge carrier properties of, in particular, single-walled carbon nanotubes from the nanoscale to the macroscale. Additionally, these nanotubes are chemically and mechanically stable, which makes them ideal for electronic components which need to be stable for a long period of time. This combination of properties appears to make them ideal components in photovoltaic solar cells. However, they also come with their own unique challenges. The most prominent of which is the electronic heterogeneity. This means that the band structure of carbon nanotubes changes with respect to their chirality, which is the angle at which a graphene sheet is rolled up into a tubular shape. Graphene has a zero-bandgap at specific symmetry points of its Brillouin zone where valence band and conduction band cross, giving it a metallic character. For one third of all unique nanotube chiralities, these symmetry points overlap with the allowed nanotube momenta, giving these carbon nanotubes the same metallic property as graphene. For the other two-thirds of chiralities, however, these symmetry points are not included in the band structure of the nanotubes. The consequence is that the bands of these nanotubes do not overlap and open a bandgap of up to around $2 \mathrm{eV}$, making them semiconducting.

This electronic heterogeneity is a challenge for integrating carbon nanotubes as components in electronic devices. Metallic nanotubes are preferable for applications which require a high conductivity such as (transparent) electrodes. Due to their bandgap semiconducting nanotubes have fewer mobile charge carriers available at room temperature and are therefore less conductive. The factor contributing most strongly to a comparatively low conductivity of a mixed carbon nanotube network is inter-tube resistance caused mainly by the formation of Schottky barriers between metallic and semiconducting nanotubes. ${ }^{[1-3]}$ In contrast, for 


\section{WILEY-VCH}

applying carbon nanotubes as charge selective contacts in solar cells, semiconducting nanotubes are preferable since they exhibit much longer charge carrier lifetimes whereas their metallic counterparts act as charge carrier recombination centers. ${ }^{[4]}$

In the initial successful approaches to integrate carbon nanotubes into photovoltaic solar cells they were employed as electron acceptors in combination with conjugated polymers in organic solar cell. ${ }^{[5,6]}$ The predominantly employed light absorbing polymer, poly(3hexylthiophene) (P3HT), should form a beneficial type-II heterojunction with single-walled carbon nanotubes, which is required to effectively dissociate excitons and to create long-lived free charges for extraction. ${ }^{[7]}$ However, devices using CNTs as electron acceptors typically yielded inexplicably low power-conversion efficiencies but extraordinarily large open-circuit voltages of up to $1.8 \mathrm{~V} \cdot{ }^{[6]}$ As an explanation it was proposed that when the polymer and the nanotubes form a junction, in the process of aligning their Fermi levels when charge carriers are exchanged, the respective electronic bands bend such that they energetically favor hole transfer from the polymer to the nanotubes. ${ }^{[8,9]}$ This was subsequently demonstrated in devices by Dabera et al., who incorporated polymer-wrapped SWNTs in an organic polymer solar cell as hole-extraction layer and thereby improving the overall performance of the device. $^{[10]}$

The p-type character of CNTs has also been exploited in junctions with n-type silicon. ${ }^{[11]}$ Such a "p-n junction” has recently been demonstrated to achieve a power-conversion efficiency of up to $17.0 \% .^{[12]}$

One of the newest additions to the family of photovoltaic solar cell systems is the perovskite solar cell. In just a few years, this class of solar cells has become a topic of intense scientific interest. $^{[13]}$ The continuous chase for even higher efficiencies has to date produced a certified efficiency of $22.1 \%{ }^{[14]}$ 


\section{WILEY-VCH}

The initial solid-state architecture was based on a layered structure with the transparent anode at the bottom, the perovskite absorber in the middle, and the hole-collecting metal cathode at the top. ${ }^{[15,16]}$ An essential part of efficient charge extraction are the charge selective contacts on both sides of the perovskite absorber. The prototypical p-type material is 2, 2', 7, 7'tetrakis(N, N-di-p-methoxy-phenylamine)-9, 9'-spirobifluorene (spiro-OMeTAD). ${ }^{[15,16]}$ The hole-transporter serves two primary functions; it blocks the transfer of electrons and facilitates the transfer of holes, which can then be extracted into the circuit. Many different holetransporting materials have been synthesized and shown to be able to efficiently extract holes. ${ }^{[17]}$

The inherent p-type nature of carbon nanotubes under ambient conditions with a workfunction in the range of 4.7-5.1 eV, ${ }^{[18-20]}$ and the successful use of carbon nanotubes as p-type contact in organic solar cells and inorganic solar cells,[10,21] makes carbon nanotubes a potential hole-transport material (HTM) for perovskite solar cells. The application of these nanotubes as HTMs may offer the benefit of being cheaper and more stable than the commonly used organic molecular or polymeric materials. The mechanical resilience of CNTs may prove to be particularly beneficial for the development of flexible perovskite solar cells. However, one of the most significant advantages of CNTs is that - in contrast to a majority of small-molecule and polymer holeconductors - carbon nanotubes would not require additional doping with dopants such as lithium salts. This dopant has been shown to compromise the stability of perovskite devices mainly due to its hygroscopic nature. Additionally the lithium-dopant is also suspected to not be stable for long-term use.[22-28] Carbon nanotubes, being inherently 


\section{WILEY-VCH}

chemically and mechanically stable and not relying on any additional dopants, have the prospect of a being a more stable alternative to conventional materials.

Making use of these properties, a range of interesting studies have been published which demonstrate various approaches to incorporating carbon nanotubes into perovskite solar cells. In the following, we will give a concise update and overview over of what we believe to be the most important ones.

In one of the first studies which employed carbon nanotubes as a charge transport element in an n-i-p mesostructured perovskite solar cells, a free-standing film of SWNTs and DWNTs was laminated onto the perovskite and used as cathode. ${ }^{[29]}$ Remarkably, the CNT film was shown to fulfill a dual-function as both hole-transporter and electrode, thus eliminating the need for a metal electrode. The comparatively low efficiency of $6.3 \%$ may be explained by relatively high resistance of the CNT film and a lack of charge-selectivity. Therefore, incorporating an additional layer of doped spiro-OMeTAD significantly improved the performance yielding a power-conversion efficiency of 9.9.\%. ${ }^{[29]}$

Multi-walled carbon nanotubes (MWNTs) were used as additional additives by Lee et al. to further improve the conductivity of doped spiro-OMeTAD. ${ }^{[30]}$ However, the authors observed a decrease in performance when the nanotubes contacted the perovskite directly, which they attributed to back-electron transfers. By inserting an addition layer of doped spiro-OMeTAD at the interface and subsequently depositing a layer of spiro-OMeTAD containing the nanotubes, they ensured the charge-selectivity of the composite hole-transport layer. The loading of $2 \mathrm{wt} \%$ of MWNTs in the spiro-OMeTAD solution improved the performance from 


\section{WILEY-VCH}

$12.8 \%$ to $15.1 \% .^{[30]}$ This improvement was attributed to a higher conductivity and reduction in series resistance.

A similar hierarchical composite structure of spiro-OMeTAD and carbon nanotubes has previously been described by us. ${ }^{[31]}$ Our initial aim in this study was to replace the lithium based dopant in spiro-OMeTAD in order to remove a potentially harmful component which is known to be highly hygroscopic and reactive. ${ }^{[27]}$ In contrast to previous studies, the n-type contact did not incorporate a mesoporous $\mathrm{TiO}_{2}$ layer instead was composed of a compact $\mathrm{TiO}_{2}$ layer and an inert alumina scaffold. ${ }^{[15]}$ Charge selectivity was therefore not exclusively reliant on the n-type contact but was enhanced by wrapping the SWNTs in a p-type polymer (P3HT) which was hypothesized to improve the charge selectivity of the SWNT film, ${ }^{[31]}$ in accordance to previous findings. ${ }^{[8,10]}$ The polymer-wrapped SWNTs were directly deposited onto the perovskite and then capped with a subsequently deposited layer of neat spiroOMeTAD. This double layer structure was shown to outperform a blend structure in which the SWNTs were randomly dispersed throughout the spiro-OMeTAD matrix. The bestperforming device reached a power-conversion efficiency of $15.4 \%$, with a series resistance as low as $1.8 \Omega / \mathrm{cm}^{2}$. Photoinduced absorption measurements were used to show that a significant fraction of photogenerated holes were transferred from the perovskite to the SWNTs. Comparing the photovoltage decay of two devices, one with just neat spiroOMeTAD and one with the double layer of SWNTs and spiro-OMeTAD, revealed that the charge extraction rate occurred by about one order of magnitude faster in the presence of the nanotubes. ${ }^{[31]}$

Based on the finding that a large fraction of photocarriers are directly injected into the SWNTs, in a follow-up study, we replaced the neat spiro-OMeTAD layer altogether by an inert polymer matrix. ${ }^{[22]}$ We thus showed that the polymer-wrapped SWNTs could operate as 


\section{WILEY-VCH}

a highly selective and effective hole-transporter in their own right, yielding a scanned powerconversion efficiency of up to $15.3 \%$. A high performance is interestingly contingent on the presence of the polymer matrix, despite it being electronically inert. While its role with regard to the charge extraction of the photogenerated holes is merely passive, the polymer layer appears to be essential to optimal device performance, by filling structural holes and gaps in the SWNT mesh-like network. This served to eliminate shunting pathways and reduce recombination between the perovskite film and the subsequently deposited metal electrode. In this structure, individual SWNTs provide a direct, high-speed connection for photogenerated holes from the perovskite to the cathode, with only few transfers required between nanotubes. It is likely that inter-tube resistances are therefore largely mitigated. ${ }^{[22]}$

Aside from preventing the metal electrode from contacting the perovskite layer, the polymer matrix has an important second role, namely improving the device stability by inhibiting the interaction between ambient moisture with the perovskite absorber. In fact, this study highlights the extreme vulnerability of the perovskite absorber towards ambient moisture, in particular, in thermal stressing experiments at an increased temperature of $80^{\circ} \mathrm{C}$. The double layer hole-transporting structure composed of polymer-wrapped SWNTs and the inert polymer matrix was shown to be able to protect the structural integrity of the perovskite while other organic hole-transporting materials could not. One of the important findings is that the hygroscopic lithium-dopant, on which most materials rely to improve their hole conductivity, accelerates moisture ingress and thus accelerating the perovskite degradation. As an aside, we do note however, that an inability to protect the perovskite layer from atmospheric moisture is not ubiquitous for all organic hole-transporters, and a later study by Leijtens et al has demonstrated very good water barrier properties from a hydrophobic organic holetransporter. $^{[32]}$

While the previous study employing CNTs had used P3HT to functionalize SWNTs, Cai et al. used carbon nanotubes to improve the charge transport characteristics of P3HT as 


\section{WILEY-VCH}

the hole-transporting material for perovskite devices. ${ }^{[33]}$ The nanotubes used in this study were a mix of single-walled and multi-walled carbon nanotubes which for structural reasons were dubbed "bamboo-like carbon nanotubes". Adding low concentrations of CNTs, in the range from $0-2 \mathrm{wt} \%$, to the $\mathrm{P} 3 \mathrm{HT}$ solution prior to deposition was investigated. The highestefficiency attained in this study was $8.3 \%$ for a CNT-loading of $1 \mathrm{wt} \%$. The observed improvement due to the presence of CNTs was ascribed to the improved crystallinity and enhanced morphology of P3HT, consistent with the findings by Ren et al. ${ }^{[34]}$ Additionally, the presence of MWNTs was linked to an increase in the conductivity of the HTL by an order of magnitude, thus reducing interfacial charge recombination. ${ }^{[35]}$ Higher loadings, however, were shown by impedance measurements to reduce the recombination resistance by opening additional charge recombination pathways. ${ }^{[33]}$ Similar findings had previously been reported by Chen et al., who had observed an improvement in efficiency from $4.1 \%$ to $6.5 \%$, by dispersing multiwalled carbon nanotubes in P3HT. ${ }^{[36]}$ The authors concluded that this improvement could be attributed to the confluence of the improved morphology and enhanced conductivity of the MWNT-P3HT matrix. ${ }^{[36]}$ In contrast, Zheng et al. were able to demonstrate that MWNTs dispersed in a very thin P3HT matrix can function as a holeselective cathode for perovskite solar cells, with a peak performance of $13.4 \% .{ }^{[37]}$ The authors compared three different approaches for their study: CNTs dispersed within P3HT (CNTs@P3HT), a layer of unfunctionalized CNTs, and a sequentially deposited double-layer of P3HT and CNTs. While all three approaches produce viable CNT-electrodes, the best performance is obtained for the network of CNTs dispersed throughout the P3HT layer. The thin P3HT matrix is thought to crosslink the MWNT network, blocking electron transfers from the perovskite and thus curbing recombination. Furthermore, the authors observe a significant improvement in the overall stability of the devices with this electrode configuration which they attribute to the hydrophobicity of $\mathrm{P} \mathrm{HT}{ }^{[37]}$ 


\section{WILEY-VCH}

Wei et al. followed the approach which had been previously demonstrated for carbon black, ${ }^{[38]}$ using MWNTs as hole-selective electrode. ${ }^{[39]}$ The MWNT film is deposited prior to the perovskite formation, thus embedding the MWNTs into the perovskite and achieving a good perovskite-nanotube interface (Figure 2 a). The comparison between carbon black and graphite flakes reveals that the superior interfacial morphology and higher conductivity of the MWNT film directly correlates with a better performance. Remarkably, in this approach neither another hole-transporting material nor a metal electrode are required for achieving devices with an efficiency of $12.7 \%$, and an impressive fill factor of 0.80 for a masked active area of $0.093 \mathrm{~cm}^{2}$ (Figure 2 c). Interestingly, the efficient hole-extraction of the MWNT layer resulted in an almost complete mitigation of the commonly observed current-voltage hysteresis. ${ }^{[39]}$ A similar device structure in which no additional hole-transporter or metal electrode is needed, was chosen by Cao et al. who investigated the SWNT loading of a holecollecting carbon electrode composed of graphite and carbon black. ${ }^{[40]}$ This study showed that the addition of $0.05 w t \%$ SWNTs to the carbon composite can improve the device performance from around $9.9 \%$ to up to $14.7 \%$, mainly by improving the photovoltage and the fill factor. ${ }^{[40]}$

Aitola et al. fabricated a hybrid carbon electrode based on single-walled carbon nanotubes and drop-cast spiro-OMeTAD (Figure 2 b). ${ }^{[41]}$ In contrast, to previous studies which had been done on methylammonium lead triiodide perovskite, the authors made use of a mixed formamidinium lead iodide-methylammonium lead bromide $\mathrm{FA}_{0.85} \mathrm{MA}_{0.15} \mathrm{~Pb}\left(\mathrm{I}_{0.85} \mathrm{Br}_{0}{ }_{15}\right)_{3}$ perovskite which yields a power-conversion efficiency of up to $18.8 \%$ for a spiro-OMeTADbased device with a gold electrode. The best-performing device with a nanotube-spiroOMeTAD composite as hole-accepting electrode, yielded a scanned efficiency of $15.5 \%$, significantly outperforming a pure SWNT electrode and a non-selective gold electrode (Figure 2 d). ${ }^{[41]}$ 


\section{WILEY-VCH}

A study by Jeon et al. investigated several avenues to integrate single-walled carbon nanotubes as electrode in place of indium-tin oxide (ITO) as the transparent cathode in the inverted p-i-n architecture. ${ }^{[42]}$ Moderate performance was achieved with a combination of SWNTs and PEDOT:PSS as electron blocking layer. In contrast, using $\mathrm{MoO}_{\mathrm{x}}$ resulted in very poor device performance. The highest efficiency (5.4\%) of an ITO-free flexible device was obtained with nitric acid as p-dopant for the SWNTs and, interestingly, without any additional electron-blocking layer. ${ }^{[42]}$ The limitations of this device, compared to a control device with ITO as electrode, were attributed to a mismatch between the energy levels of the SWNTs and the perovskite absorber. ${ }^{[42]}$

Two studies coming out of the National Renewable Energy Laboratory investigated in detail more fundamental questions regarding the perovskite-nanotube interface. ${ }^{[43,44]}$ Purified semiconducting SWNTs with a narrow $(6,5)$ chirality were deposited, layer-by-layer, onto a perovskite layer and probed by photoemission spectroscopy. Schulz et al. could thus show that upon contact, electron donation from the perovskite to the nanotubes occurs. This effectively n-dopes the nanotubes, causing them to undergo significant downwards band bending towards the interface, with a magnitude of over $0.5 \mathrm{eV}$ (Figure $3 \mathbf{~ a )}{ }^{\left[{ }^{43]}\right.}$

By probing the interface with XPS measurements, the authors could also show that the ndoping occurs as a result of electron transfer from the methylammonium cation in the perovskite to the SWNTs. Transient absorption spectroscopy measurements confirmed the beneficial effect of the band bending by showing that holes are extracted by the nanotubes on an even faster time scale than the concomitantly photogenerated electrons are extracted by the $\mathrm{TiO}_{2}$, which forms the n-type contact. ${ }^{[43]}$ The band bending is credited with facilitating rapid 


\section{WILEY-VCH}

transfer of photogenerated holes away from the interface and hindering back-transfer of holes thus reducing recombination losses.

Ihly et al. followed-up on the previous study by carefully probing the charge transfer kinetics between the perovskite and SWNTs using time-resolved spectroscopy over time scales spanning from femtoseconds to several hundreds of microseconds. ${ }^{[44]}$ By using a highly purified distribution of semiconducting monochiral SWNTs, the authors were able to unequivocally assign changes in the absorption spectra to the excitonic SWNT transition.

Femtosecond transient-absorption measurements show that charge extraction of photogenerated holes sets in within the first picosecond after generation by the laser pulse, and is completed after three nanoseconds (Figure $\mathbf{3} \mathbf{b}$ ). The transient absorption dynamics reveal further that neither $\mathrm{TiO}_{2}$ as n-type contact, nor spiro-OMeTAD as p-type contact markedly change the bleach recovery time. In contrast, SWNTs at the interface significantly reduce the bleach recovery time. This shows that the SWNTs are able to remove photogenerated holes from the perovskite absorber faster than spiro-OMeTAD. Once injected into the SWNTs, these holes are also shown to be extremely long-lived, several orders of magnitude longer than in the perovskite (Figure $3 \mathbf{c}$ ). This indicates that the previously shown potential barrier due to band bending, prevents back-transfer or recombination of the holes.

In this study, time-resolved microwave conductivity measurements are used to show that the efficient hole extraction improves the extraction of electrons by $\mathrm{TiO}_{2}$. The authors suggest that the rapid removal of holes inhibits recombination and thus raises the quasi-Fermi level of the electrons in the perovskite, which would increase the driving force for the previously poor electron injection into the $\mathrm{TiO}_{2}$. Finally, the enhanced charge extraction of the SWNTs is shown by employing a thin $(5 \mathrm{~nm})$ SWNT film as interfacial layer between the perovskite and 


\section{WILEY-VCH}

doped spiro-OMeTAD, and thus improving the power-conversion efficiency from $14.7 \%$ to $16.5 \% .^{[44]}$

One of the first, very interesting, applications which takes advantage of the unique set of properties of carbon nanotubes, namely their excellent charge transport characteristics as well as their inherent mechanical flexibility, are flexible perovskite solar cell fibers which have

been pioneered by the group of Huisheng Peng. ${ }^{[45-47]}$ With the aim to create energy harvesting textiles, the researchers have fabricated coaxial solar cells, in which the perovskite absorber is sandwiched between an n-type core wire and the flexible p-type nanotubes (Figure $3 \mathbf{d}$ ). The cathode forming the outermost layer is a sheet of aligned carbon nanotubes which were drydrawn from a spinnable array. The resulting devices are shown to be operational at around $90 \%$ of their initial power-conversion efficiency after 250 stretch cycles. ${ }^{[46]}$ The efficiency of these devices has thus far not exceeded $10 \%$, but for a PV fabric, this is very interesting.

\section{Conclusion}

In summary, several very promising approaches which utilize the charge properties of carbon nanotubes to extract photogenerated holes in perovskite devices have been reported. The exact function of the nanotubes in different architectures ranges from hole-transporter additive and interface modifier, to hole-transporting system to charge-selective electrode. Their inherent resilience and stability appears to be one of their strongest advantages over other materials which can play the same roles. Further improvements in the power-conversion efficiency using carbon nanotube based device components could make them a highly competitive, and advantageous option for high-performing devices with long-term stability; two components which will be required for the commercialization of this technology. 


\section{WILEY-VCH}

\section{Acknowledgements}

This work was supported by the International Collaborative Energy Technology R\&D Program of the Korean Institute of Energy Technology Evaluation and Planning (KETEP) with financial resource from the Ministry of Trade, Industry \& Energy, Republic of Korea (no. 20148520011250)

[1] Z. Yao, C. Dekker, H. W. C. Postma, L. Balents, Nature 1999, 402, 273.

[2] M. S. Fuhrer, J. Nygård, L. Shih, M. Forero, Y.-G. Yoon, H. J. Choi, J. Ihm, S. G. Louie, A. Zettl, P. L. McEuen, Science 2000, 288, 494.

[3] P. N. Nirmalraj, P. E. Lyons, S. De, J. N. Coleman, J. J. Boland, Nano Lett. 2009, 9, 3890.

[4] J. M. Holt, A. J. Ferguson, N. Kopidakis, B. A. Larsen, J. Bult, G. Rumbles, J. L. Blackburn, Nano Lett. 2010, 10, 4627.

[5] E. Kymakis, G. A. J. Amaratunga, Rev. Adv. Mater. Sci. 2005, 10, 300.

[6] R. L. Patyk, B. S. Lomba, A. F. Nogueira, C. A. Furtado, A. P. Santos, R. M. Q. Mello, L. Micaroni, I. A. Hümmelgen, Phys. Status Solidi - Rapid Res. Lett. 2007, 1, 43.

[7] T. Schuettfort, A. Nish, R. J. Nicholas, Nano Lett. 2009, 9, 3871.

[8] N. M. Dissanayake, Z. Zhong, Nano Lett. 2011, 11, 286.

[9] F. Lan, G. Li, Nano Lett. 2013, 13, 2086.

[10] G. D. M. R. Dabera, K. D. G. I. Jayawardena, M. R. R. Prabhath, I. Yahya, Y. Y. Tan, N. A. Nismy, H. Shiozawa, M. Sauer, G. Ruiz-Soria, P. Ayala, V. Stolojan, a a D. T. Adikaari, P. D. Jarowski, T. Pichler, S. R. P. Silva, ACS Nano 2013, 7, 556.

[11] X. Li, Z. Lv, H. Zhu, Adv. Mater. 2015, 27, 6549. 


\section{WILEY-VCH}

[12] F. Wang, D. Kozawa, Y. Miyauchi, K. Hiraoka, S. Mouri, Y. Ohno, K. Matsuda, Nat. Commun. 2015, 6, 6305.

[13] M. a. Green, T. Bein, Nat. Mater. 2015, 2.

[14] National Renewable Energy Laboratory, "NREL Efficiency Chart,” can be found under http://www.nrel.gov/ncpv/images/efficiency_chart.jpg, 2016.

[15] M. M. Lee, J. Teuscher, T. Miyasaka, T. N. Murakami, H. J. Snaith, Science 2012, 338, 643.

[16] H.-S. Kim, C.-R. Lee, J.-H. Im, K.-B. Lee, T. Moehl, A. Marchioro, S.-J. Moon, R. Humphry-Baker, J.-H. Yum, J. E. Moser, M. Grätzel, N.-G. Park, Sci. Rep. 2012, 2, 591.

[17] Z. Yu, L. Sun, Adv. Energy Mater. 2015, 5, 1500213.

[18] Collins, Bradley, Ishigami, Zettl, Science 2000, 287, 1801.

[19] M. Shiraishi, M. Ata, Carbon N. Y. 2001, 39, 1913.

[20] P. Liu, Q. Sun, F. Zhu, K. Liu, K. Jiang, L. Liu, Q. Li, S. Fan, Nano Lett. 2008, 8, 647.

[21] Y. Jung, X. Li, N. K. Rajan, A. D. Taylor, M. a Reed, Nano Lett. 2013, 13, 95.

[22] S. N. Habisreutinger, T. Leijtens, G. E. Eperon, S. D. Stranks, R. J. Nicholas, H. J. Snaith, Nano Lett. 2014, 14, 5561.

[23] J. Yang, B. D. Siempelkamp, D. Liu, T. L. Kelly, ACS Nano 2015, 9, 1955.

[24] Y. S. Kwon, J. Lim, H.-J. Yun, Y.-H. Kim, T. Park, Energy Environ. Sci. 2014, 7, 1454.

[25] J. Liu, S. Pathak, T. Stergiopoulos, T. Leijtens, K. Wojciechowski, S. Schumann, N. Kausch-Busies, H. J. Snaith, J. Phys. Chem. Lett. 2015, 1666.

[26] A. Abate, D. J. Hollman, J. Teuscher, S. Pathak, R. Avolio, G. D’Errico, G. Vitiello, S. Fantacci, H. J. Snaith, J. Am. Chem. Soc. 2013, 135, 13538.

[27] A. Abate, T. Leijtens, S. Pathak, J. Teuscher, R. Avolio, M. E. Errico, J. Kirkpatrik, J. M. Ball, P. Docampo, I. McPherson, H. J. Snaith, Phys. Chem. Chem. Phys. 2013, 15, 


\section{WILEY-VCH}

2572.

[28] W. H. Nguyen, C. D. Bailie, E. L. Unger, M. D. McGehee, J. Am. Chem. Soc. 2014, 136, 10996.

[29] Z. Li, S. a Kulkarni, P. P. Boix, E. Shi, A. Cao, K. Fu, S. K. Batabyal, J. Zhang, Q. Xiong, L. H. Wong, N. Mathews, S. G. Mhaisalkar, ACS Nano 2014, 8, 6797.

[30] J. Lee, M. M. Menamparambath, J.-Y. Hwang, S. Baik, ChemSusChem 2015, 8, 2358.

[31] S. N. Habisreutinger, T. Leijtens, G. E. Eperon, S. D. Stranks, R. J. Nicholas, H. J. Snaith, J. Phys. Chem. Lett. 2014, 5, 4207.

[32] T. Leijtens, T. Giovenzana, S. N. Habisreutinger, J. S. Tinkham, N. K. Noel, B. A. Kamino, G. Sadoughi, A. Sellinger, H. J. Snaith, ACS Appl. Mater. Interfaces 2016, 8, 5981.

[33] M. Cai, V. T. Tiong, T. Hreid, J. Bell, H. Wang, J. Mater. Chem. A Mater. energy Sustain. 2014, 00, 1.

[34] S. Ren, M. Bernardi, R. R. Lunt, V. Bulovic, J. C. Grossman, S. Gradečak, Nano Lett. 2011, 11, 5316.

[35] A. W. Musumeci, G. G. Silva, J. W. Liu, W. N. Martens, E. R. Waclawik, Polymer (Guildf). 2007, 48, 1667.

[36] H. Chen, X. Pan, W. Liu, M. Cai, D. Kou, Z. Huo, X. Fang, S. Dai, Chem. Commun. (Camb). 2013, 49, 7277.

[37] X. Zheng, H. Chen, Z. Wei, Y. Yang, H. Lin, S. Yang, Front. Optoelectron. 2016, 1.

[38] A. Mei, X. Li, L. Liu, Z. Ku, T. Liu, Y. Rong, M. Xu, M. Hu, J. Chen, Y. Yang, M. Grätzel, H. Han, Sci. 2014, 345 , 295.

[39] Z. Wei, H. Chen, K. Yan, X. Zheng, S. Yang, J. Mater. Chem. A 2015, 3, 24226.

[40] H. Li, K. Cao, J. Cui, S. Liu, X. Qiao, Y. Shen, M. Wang, Nanoscale 2016, DOI 10.1039/C5NR07347B.

[41] K. Aitola, K. Sveinbjornsson, J. P. Correa Baena, A. Kaskela, A. Abate, Y. Tian, E. M. 


\section{WILEY-VCH}

J. Johansson, M. Grätzel, E. Kauppinen, A. Hagfeldt, G. Boschloo, Energy Environ. Sci. 2015, 461.

[42] I. Jeon, T. Chiba, C. Delacou, Y. Guo, A. Kaskela, O. Reynaud, E. I. Kauppinen, S. Maruyama, Y. Matsuo, Nano Lett. 2015, 15, 6665.

[43] P. Schulz, A.-M. Dowgiallo, M. Yang, K. Zhu, J. L. Blackburn, J. J. Berry, J. Phys. Chem. Lett. 2016, 7, 418.

[44] R. Ihly, A.-M. Dowgiallo, M. Yang, P. Schulz, N. Stanton, O. G. Reid, A. Ferguson, K. Zhu, J. J. Berry, J. Blackburn, Energy Environ. Sci. 2016, DOI 10.1039/C5EE03806E.

[45] L. Qiu, J. Deng, X. Lu, Z. Yang, H. Peng, Angew. Chemie - Int. Ed. 2014, 53, 10425.

[46] J. Deng, L. Qiu, X. Lu, Z. Yang, G. Guan, Z. Zhang, H. Peng, J. Mater. Chem. A 2015, 3, 21070.

[47] L. Qiu, S. He, J. Yang, J. Deng, H. Peng, Small 2016, 2419.

Figure 1. In panels a) and b) the schematics of two of the first publications in which carbon nanotubes are employed in perovskite solar cells. The device in a) uses a laminated CNT film as a electrode without dedicated hole-transport material and is shown to perform even better than a control device with a gold electrode (panel c). In the architecture in b) a composite structure of functionalized SWNT and an inert polymer matrix constitutes the hole-selective charge transport layer, which yields a performance of up to $15.3 \%$ (panel d). Reproduced and adapted with permission. ${ }^{[22,29]}$ Copyright 2014, American Chemical Society. 
a)
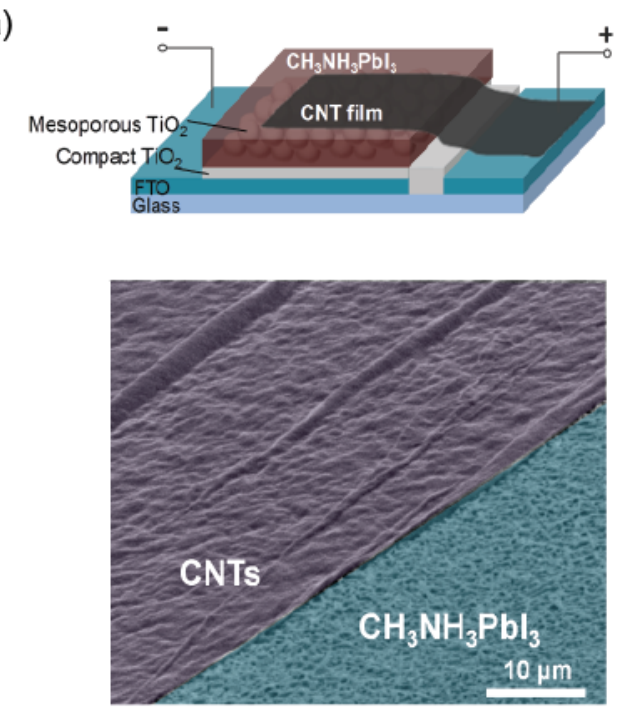

c)

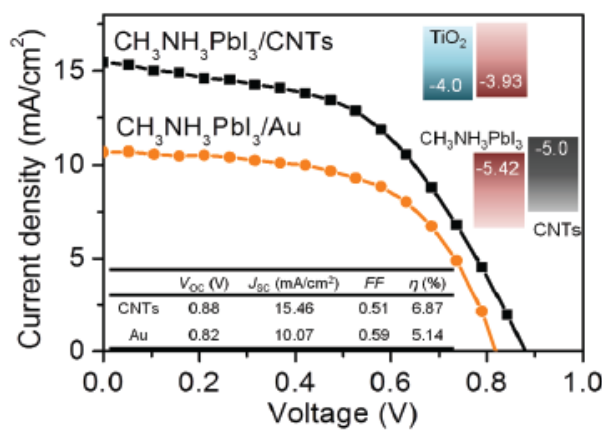

b)

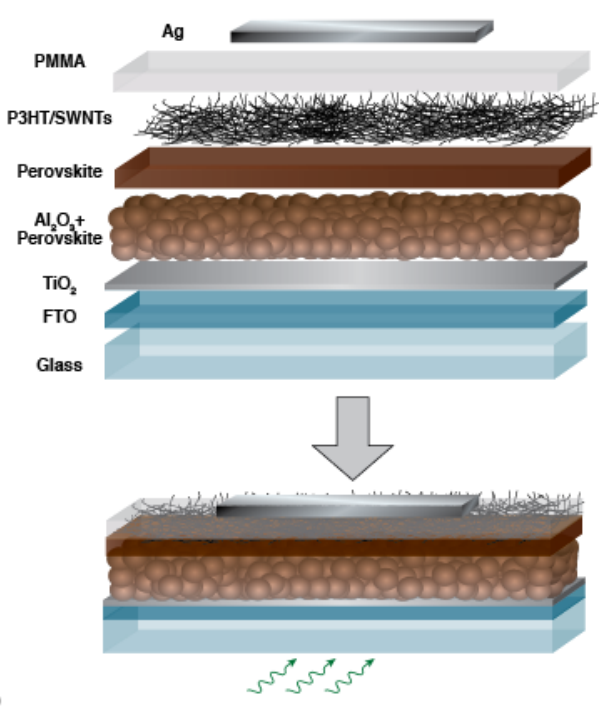

d)

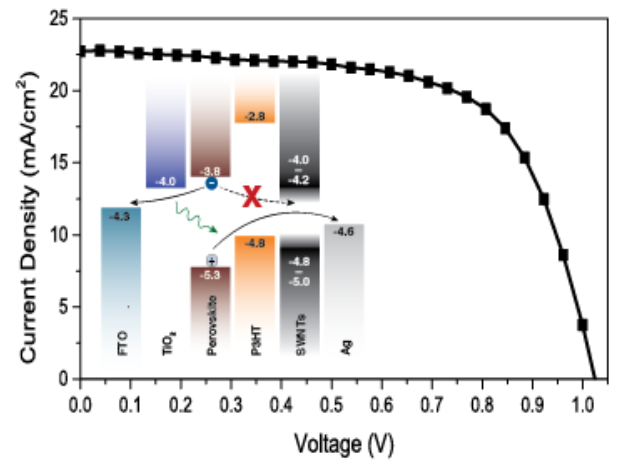

Figure 2: In a) MWNTs are incorporated as hole-selective electrode into a perovskite solar cell by depositing them onto $\mathrm{PbI}_{2}$ before converting it to $\mathrm{MAPbI}_{3}$ through MAI conversion. The embedded MWNT network has therefore excellent, continuous contact with the perovskite layer. As a result the devices exhibit fill factors of up to 0.80 (panel c) and appear to be hysteresis free. Reproduced and adapted with permission. ${ }^{\text {[39] }}$ Copyright 2015, The Royal Society of Chemistry. b) shows the fabrication sequence of a hybrid hole-selective electrode on a mixed perovskite absorber composed of SWNTs and spiro-OMeTAD. In d) the current-voltage characteristics of this structure is compared to spiro-OMeTAD+gold, SWNTs and gold, showing that the combination of SWNTs and spiro-OMeTAD improves device performance compared to just SWNTs and gold, but is not quite yet on par with spiro-OMeTAD and gold combined. 


\section{WILEY-VCH}

Reproduced with permission. ${ }^{[41]}$ Copyright 2016 The Royal Society of Chemistry.

a)
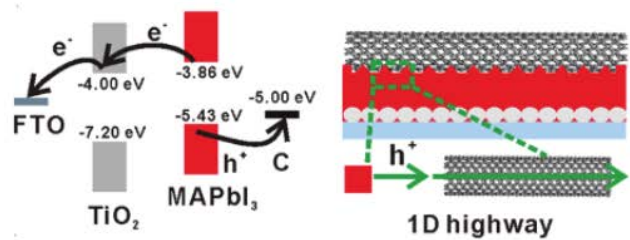

c)

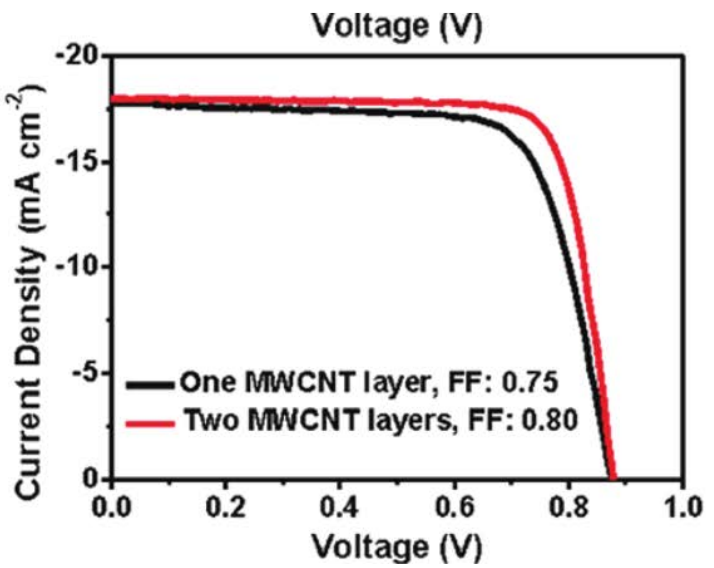

b)

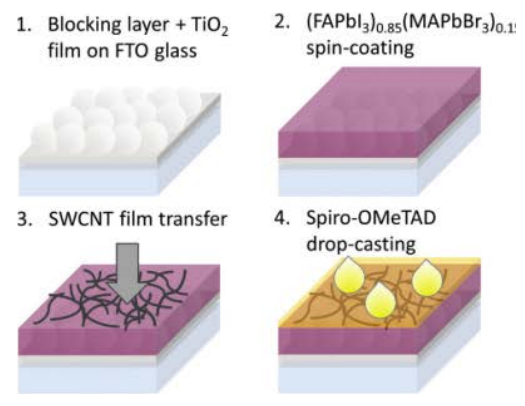

d)

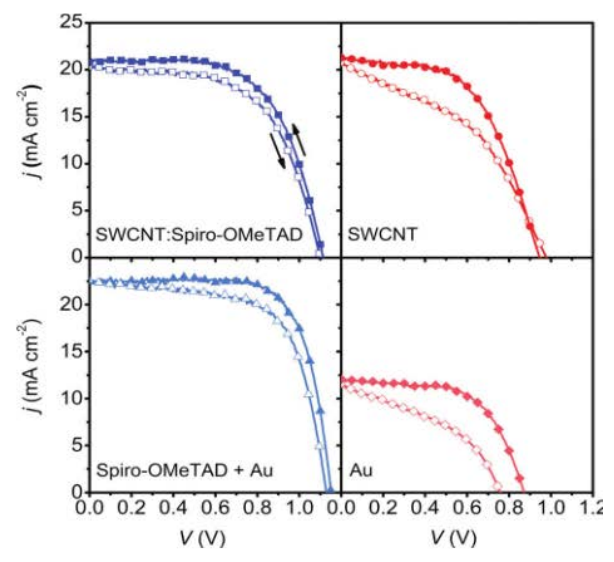

Figure 3. Depositing sequential layers of $2 \mathrm{~nm}$ of purified single-chirality SWNTs onto $\mathrm{MAPbI}_{3}$ and probing them by photoemission spectroscopy, Schulz et al. could demonstrate that charge transfer between the nanotubes and the perovskite absorber results in an n-type doping of the SWNTs, shown in a). Reproduced with permission. ${ }^{[43]}$ Copyright 2016 American Chemical Society. Employing femtosecond transient-absorption, photogenerated holes are shown to be extracted at the SWNTperovskite interface within the first picosecond after generation by the laser pulse, and is completed after three nanosecond b). After the very fast charge transfer into the SWNTs, the holes are shown to be very long lived (panel c). Reproduced with permission. ${ }^{[44]}$ Copyright 2016 The Royal Society of Chemistry. In d) the integration of a sheet of CNTs as flexible p-type electrode into a fiber is shown. Reproduced with 
permission. ${ }^{[45]}$ Copyright 2014 Wiley-VCH Verlag GmbH.

a)
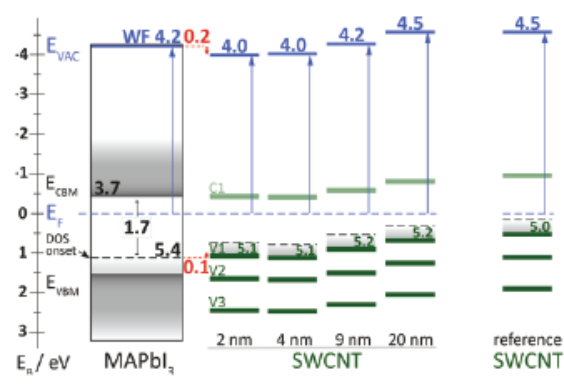

c)

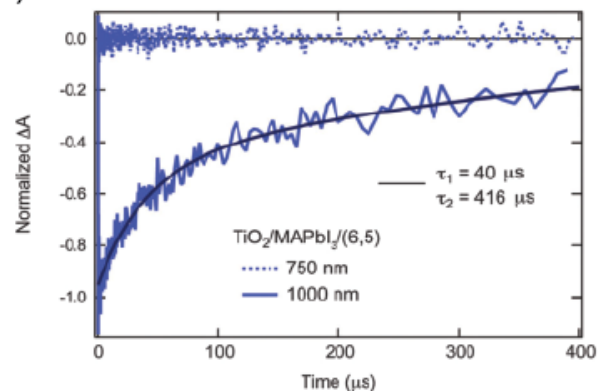

b)

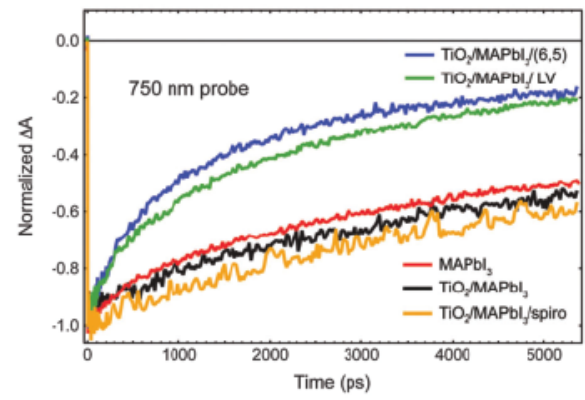

d)

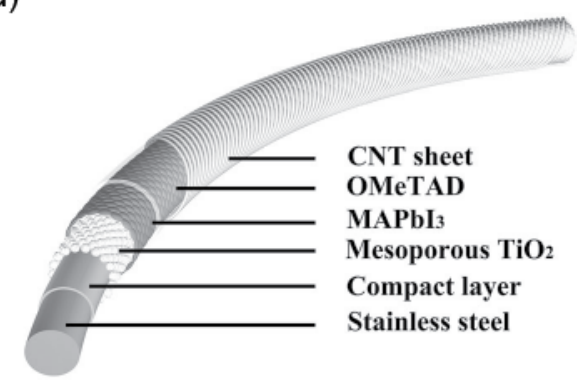

Received: ((will be filled in by the editorial staff))

Revised: ((will be filled in by the editorial staff)) Published online: ((will be filled in by the editorial staff)) 


\section{WILEY-VCH}

The table of contents entry should be 50-60 words long, and the first phrase should be bold. The entry should be written in the present tense and impersonal style. The text should be different from the abstract text.

\section{Keyword}

perovskite solar cells, single-walled carbon nanotubes, multi-walled carbon nanotubes, carbon nanotubes

Severin N. Habisreutinger, Robin J. Nicholas*, Henry J. Snaith* ((same order as byline))

\section{Title}

Carbon nanotubes in perovskite solar cells 